\title{
Evaluating perception in driving simulation experiments
}

\author{
Andras Kemeny ${ }^{1,2}$ and Francesco Panerai ${ }^{1}$ \\ ${ }^{1}$ Laboratoire de Physiologie de la Perception et de I'Action, CNRS-Collège de France, 11, Place M. Berthelot, 75005 Paris, France \\ ${ }^{2}$ Technical Centre for Simulation, Renault Technocentre, 1 avenue du Golf, 78288 Guyancourt Cedex, France
}

\begin{abstract}
The use of driving simulation for vehicle design and driver perception studies is expanding rapidly. This is largely because simulation saves engineering time and costs, and can be used for studies of road and traffic safety. How applicable driving simulation is to the real world is unclear however, because analyses of perceptual criteria carried out in driving simulation experiments are controversial. On the one hand, recent data suggest that, in driving simulators with a large field of view, longitudinal speed can be estimated correctly from visual information. On the other hand, recent psychophysical studies have revealed an unexpectedly important contribution of vestibular cues in distance perception and steering, prompting a re-evaluation of the role of visuo-vestibular interaction in driving simulation studies.
\end{abstract}

Vehicle driving implies perception and control of selfmotion at a greater range of velocities than locomotion by walking. It is often considered to be a task dominated by visual information. However, it is well-established that other sensory information, such as that provided by the vestibular and PROPRIOCEPTIVE (see Glossary) channels, also contributes to the perception and control of selfmotion. Motivated by a recent renewed interest in the role and function of these non-visual sensory modalities, we aim in this review to re-evaluate the role of visuovestibular interactions in driving simulation experiments, and to assess how applicable driving simulation is to the real world for studies of vehicle dynamics or driver behaviour.

In 1938 Gibson [1] proposed a psychophysical theory of perception for automobile-driving, defining a 'terrain of field of space' for the driver, with the car considered as a tool of locomotion and the driver aiming to drive in the middle of a 'field of safe travel'. In 1950 he described the visual perception of space [2] based on visual depth, distance or orientation stimulus variables. OPTIC FLOW, one of the most important visual cues he proposed, is defined as the visual motion experienced as a result of walking or driving, and it is thought to play a dominant role in the control of heading [3] and collision detection [4-7]. However, regarding the control of the direction of movement in natural environments (i.e. walking), there is still disagreement over whether the structure of the flow
[8,9] or the visual EGOCENTRIC DIRECTION per se $[10,11]$ is the dominant source of information. It is not clear either whether the same strategies used for natural locomotion apply to driving situations where displacements occur at higher velocities. Interestingly, a new point of view on these controversial issues was recently provided by experiments performed in driving simulators [12]. However, Gibson's original theory also included a definition of the perceptual field of the car itself, bringing to the driver kinaesthetic and tactile cues. These ideas were applied to driving simulation from the early 1980s [13-15], and since then many simulator experiments have been carried out for vehicle design [16-18] and driver perception studies $[19,20]$.

Driving simulators provide most, but not all of the relevant visual cues present when driving in the real world. Importantly, optic flow resulting from the continuous movement of the textured images of all objects in the scene, is present (see Box 1). However, binocular cues, as well as MOTION PARALLAX due to the observer's head movement, are often absent in simulators. Their presence would increase the complexity, and cost, of image generation and display equipment, and would necessitate the integration of head tracking devices. Visual cues are rendered by real-time generation of 3-D images of the surrounding landscape, corresponding to the driven vehicle position in the virtual world (Box 1). The precise role of each cue for perception needs to be respected to provide a coherent representation of the world, crucial for any moving observer, but especially one driving an automobile.

\section{Glossary}

Disparity: the relative lateral displacement of the retinal images in the left and right eyes of the same object in space. It is an effective binocular cue to depth at short distances.

Egocentric direction: the direction of an object in space relative to the observer. Egocentric direction is determined by retinal position, proprioceptive information about the eye, head and body position.

Haptic perception: involves both tactile perception through the skin and kinaesthetic perception of the position and movement of the joints and muscles.

Motion parallax: the differential motion of pairs of points as a result of their different depths relative to the fixation point and to the motion of the observer. (See Box 2).

Optic flow: the dynamic pattern of information available in the optic array along a moving trajectory of viewpoints.

Proprioceptive cues: information about the state of the body's motion and posture, as signaled by various systems, such as the muscle spindles, vestibular organs and including some visual components. 


\section{Box 1. Visual and motion cueing in driving simulation}

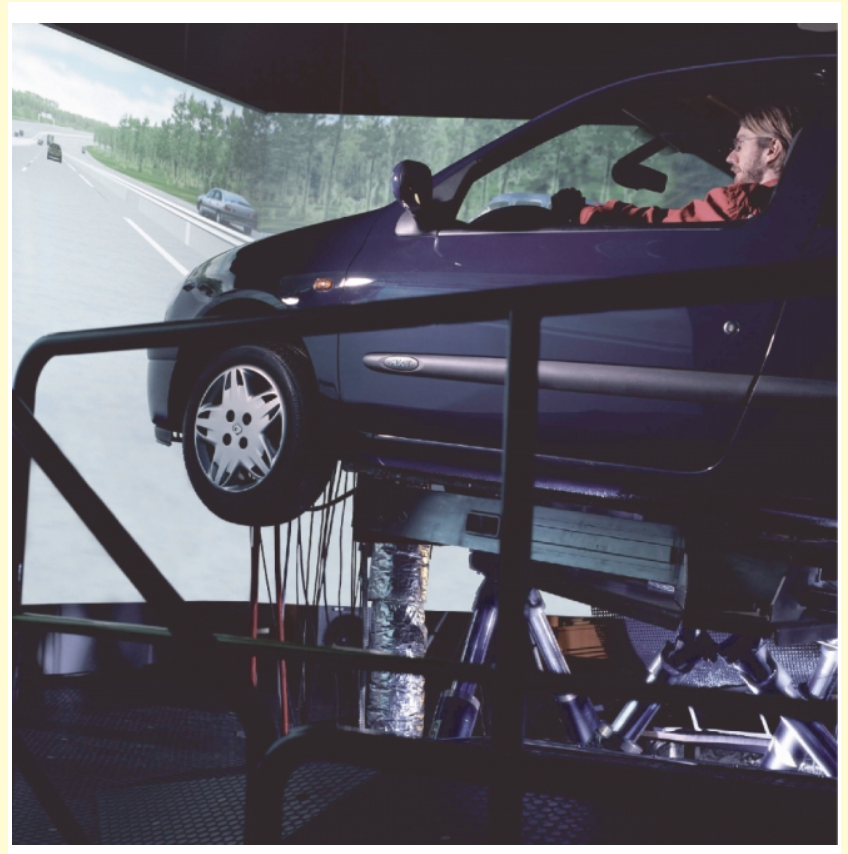

TRENDS in Cognitive Sciences

Fig. I. The driving simulator at Renault's Technocentre uses a real vehicle mounted on a six-axis motion platform that allows 3-D vehicle movement. To simulate a lane change, the platform shifts laterally with appropriate dynamics, giving the driver the perception of a lateral acceleration. Similarly, for sustained braking, longitudinal deceleration is simulated by having the platform modify the vehicle's angular attitude (tilt).

A driving simulator is a system providing a coherent multi-sensory environment for a driver to perceive and control virtual vehicle movements. The driver sits in a cockpit and activates commands (see Fig. I). These determine the simulated vehicle motion on the basis of a vehicle dynamic model. Driver's commands and head or eye movements, vehicle position and orientation, traffic information when available, as well as other physiological measurements are recorded during the simulation session for driver behavior analysis (see Fig. II).

\section{Visual cueing}

Visual cues are provided by an image generator, which computes in real-time the textured images of the simulated scenes. Generally, these are projected on a curved screen or one or more flat screens. Some simulators use head-mounted displays (HMD). Such configurations usually provide stereoscopic viewing and head movement tracking. However, the field of view is generally limited. It has been found that for correct speed perception, a horizontal field of view of at least $120^{\circ}$ is needed [a]. Although linear perspective, texture mapping and lighting are provided by most state-of-the-art driving simulators, parallax due to driver head movements, and stereopsis are rarely found, with the exception of HMD-based installations. Whereas it is generally accepted that the effectiveness of binocular convergence as a cue to absolute distance is limited to a few meters [b], the effectiveness of binocular disparity is judged to be up to $\sim 30 \mathrm{~m}$, although this is still controversial [c]. Recent results in visual psychophysics [d] suggest that motion parallax due to observer movement can contribute to improve depth perception in driving simulation experiments.

\section{Motion cueing}

Several studies provide evidence that vestibular cues have a role in steering and speed control $[\mathrm{e}-\mathrm{f}]$. Motion cueing can be obtained thanks to a movement platform which is controlled by a set of six electromechanical linear actuators mounted in a hexapod configuration, also known as a Stewart Platform (seen in Fig. I). It generates linear acceleration in the longitudinal, lateral and vertical direction of the vehicle, as well as roll, pitch and yaw angular accelerations. To extend the range of physical movement, a large linear actuator can be added to the Stewart platform in the longitudinal and lateral direction. Driver simulator validity is an on-going field of investigation [g]. It has been shown that humans accept a great deal of variation in perceived vestibular linear and rotational acceleration amplitudes [h], as well as in the temporal integration of visual and vestibular cues while driving [i-j]. Consequently some authors suggest the use of scale factors for the rendering of motion cueing, in order for it to be realistic even with limited displacements [k].

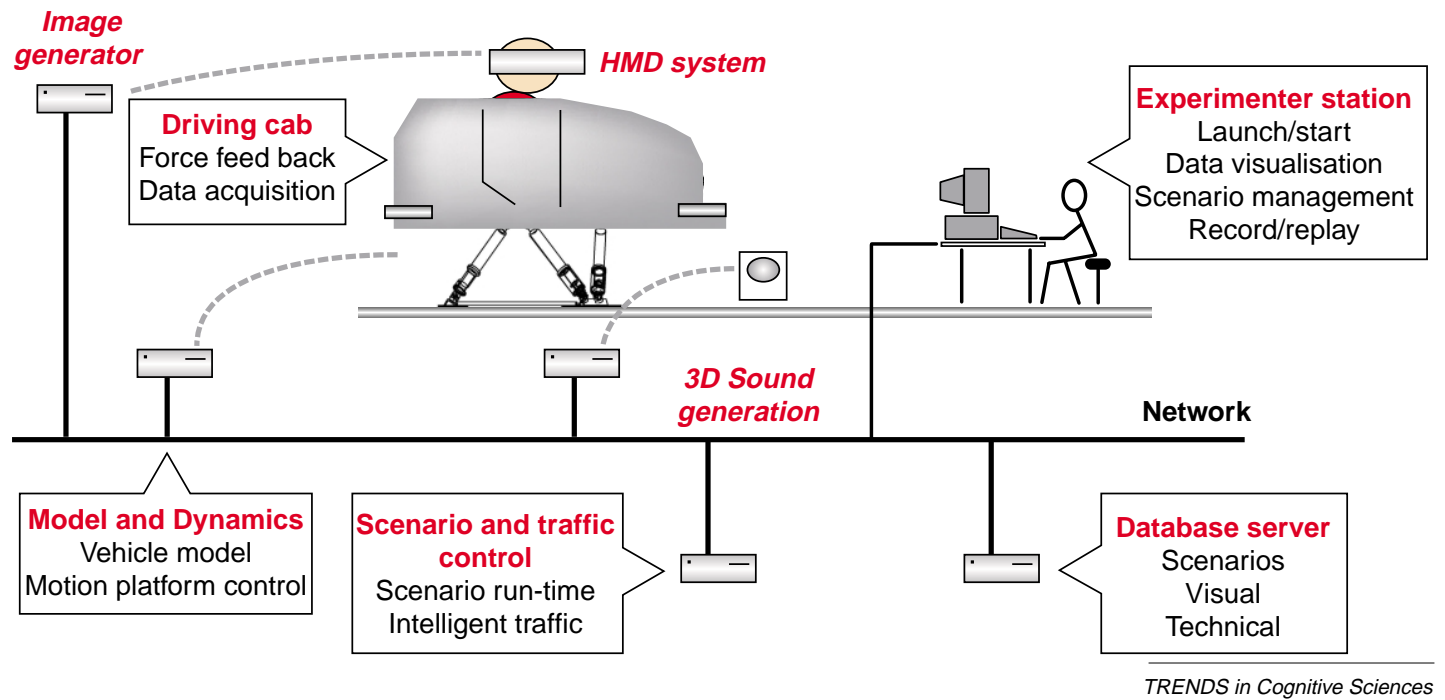

Fig. II. The architecture of a driving simulator using a head-mounted display (HMD). A multi-processor architecture, distributed across a network of computers, enables the generation of coherent images comprising both sound and motion stimuli. A separate database server dispatches all the information concerning the simulated scenario across the network of computers. 


\section{References}

a Jamson, H (2000) Driving simulation validity: issues of field of view and resolution. Proc. Driving Simul. Conf. Paris, France, pp. $57-64$

b von Hofsten, C. (1976) The role of convergence in visual space perception. Vis. Res. 16, 193-198

c Loomis, J.M. and Knapp, J.M. (1999) Visual perception of egocentric distance in real and virtual environments. Virtual and Adaptive Environments (Hettinger, L.J., Haas, M.W. eds), Erlbaum

d Peh, C.H. et al. (2002) Absolute distance perception during in-depth head movement: calibrating optic flow with extra-retinal information. Vis. Res. 42, 1991-2003

e Reymond, G. et al. (2001) Role of lateral acceleration in curve driving: driver model and experiments on a real vehicle and a driving simulator. Hum. Factors 43, 483-495

$\mathrm{f}$ Wierwille, W.W. et al. (1983) Driver steering reaction time to abrupt-onset crosswinds, as measured in a moving-base driving simulator. Hum. Factors 25, 103-116

g Reymond, G. and Kemeny, A. (2000) Motion cueing in the Renault driving simulator. Veh. Syst. Dynam. 34, 249-259

h van der Steen, F.A. (1998) An earth-stationary perceived visual scene during roll and yaw motions in a flight simulator. J. Vestib. Res. 8, 411-425

i Cunningham, D.W. et al. (2001) Driving in the future: temporal visuomotor adaptation and generalization. J. Vision 1, 88-98

j Dagdelen, M. et al. (2002) Analysis of the visual compensation in the Renault driving simulator. Proc. Driving Simul. Conf. Paris, France, pp. 109-119

k Groen, E.L. et al. (2000) Psychophysical thresholds associated with the simulation of linear acceleration. Am. Inst. Aeronaut. Astronaut. 1, 4294-4302

\section{Simulation fidelity}

An important and often underestimated issue is simulation fidelity. When driving tasks are not the main focus of an experiment, relative perceptual fidelity, permitting only certain types of comparisons between the simulated and the real world, might be acceptable. Training, general dashboard ergonomics and driver alertness studies are examples of such driving simulation experiments, where partial simulator configurations can be efficiently used for vehicle applications or human factor studies. By contrast, absolute simulation fidelity is needed when driver behaviour is studied as a function of road, visibility conditions, vehicle or traffic characteristics. Such studies often require a careful analysis of the complete set of perceptual variables used by the driver. For example, the correct perception of 'time-headway' - the time interval between two vehicles following one another - is crucial for studies aiming to analyse driver behaviour when using adaptive cruise-control systems that regulate inter-vehicle distance.

The kind of display equipment used can also affect simulation fidelity. For example, a recent experiment that involved displaying computer generated images of the road environment on a computer monitor, suggested drivers' speed perception is greatly reduced in foggy conditions [21]. However, other studies show that a limited field of view induces poor perception of speed by the driver [22], so conclusions derived from computer-based simulations might be unreliable. Studies of speed perception in reduced visibility conditions that lack a large field of view during experimentation might therefore be valid only in the context of driving in poor road infrastructure

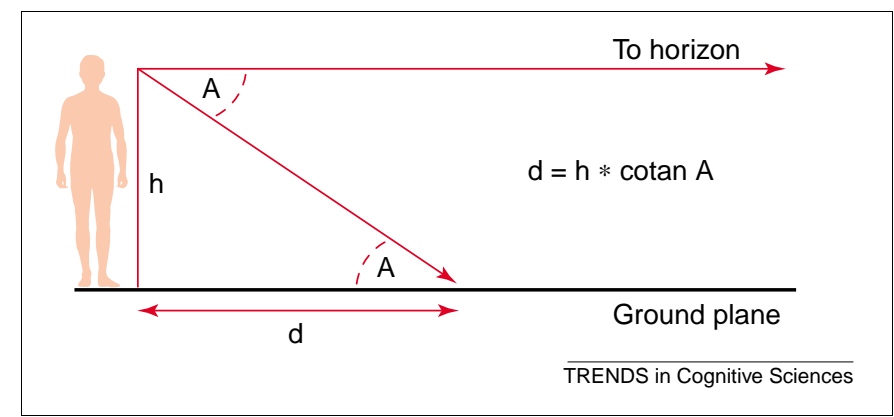

Fig. 1. Angular declination from the horizon as a cue to absolute distance. The visual system can compute absolute distance (d) to an object on the ground plane from eye height $(\mathrm{h})$ and the angular declination below the horizon (A) [24]. Mathematically, distance is given by the product of the eye-height (h) and the cotangent of the angle $(\mathrm{A})$ between the line of sight to the horizon and the ground plane point.

environments. For example, in urban areas, even under poor visibility conditions, there are many other speed perception cues in the peripheral field of view.

Another visual cue, angular declination - the horizon angle relative to a point of the ground plane (see Fig. 1) was recently shown to be a strong determinant of perceived distance. Manipulating angular declination alone causes a change in perceived distance [23,24]. For example, in an experiment we carried out in a lorry driving simulator with a large field of view [16], we varied the simulated eye-height in a task where the lorry driver should maintain a safe distance with respect to a leading car vehicle. We observed that by increasing the simulated eye-height, the corresponding perceived (safety) distance was also increased. Moreover, for the same increment in the simulated eye-height, we observed higher travelling speeds, suggesting a reduced subjective speed perception. These findings suggest, that in a driving simulator, the incorrect calibration of driver's eye height, and the consequent visually perceived eye level, might induce biased observations of inter-vehicle distances. This could lead to unreliable results from studies of cruise-control systems, for example. By contrast, a careful analysis of drivers' perception of inter-vehicle distances as a function of visually perceived eye level, might lead to safer motor vehicles and driver aid system design.

Finally, as Gibson has already pointed out, kinaesthetic cues also strongly influence the perception of speed $[1,14,19]$. To simulate vestibular stimuli, accelerations to be perceived by the driver can be rendered by real time generation of simulator cockpit motion, following appropriate vehicle dynamics. The precise role and importance of vestibular cues remains the subject of ongoing research.

\section{Speed and distance perception}

While driving, evaluation of vehicle speed and intervehicle distance are crucial skills and constant demands. Manoeuvers such as braking, obstacle avoidance and overtaking are based on such skills. From the perspective of human perception, these skills rely on the representation of (1) self-motion in the 3-D environment, and (2) the egocentric distances (i.e. distance from an observer to a target, or in driving simulation inter-vehicle distance, for example). What are the perceptual cues used by the driver 
and what is their effectiveness during driving simulation experiments?

\section{Visual cues}

Of the visual cues available during locomotion, optic flow (see glossary) has been the most extensively investigated [3]. Optic flow cannot give information about absolute distance to an object and travel speed. Rather it can be used to compare spatial intervals [25] and for time measurements relative to the object and observer- the time-to-contact [6,26] (but see also [7]). Under certain conditions, optic flow has been shown to be a reliable cue to estimate distance of travel [27,28]. On the one hand, because the driver's and environmental object's speeds determine velocities in the optic flow pattern, knowledge of road markings or other scale factors can help the driver make good estimates of speed from optic flow. On the other hand, psychophysical studies on motion perception have shown that observers can underestimate speed when image contrast [29], texture [30] or luminance [31] are reduced. These same mechanisms might lead one to underestimate driving speed in foggy weather [32] or during night driving [33]. In a study carried out in a dynamic driving simulator with a large field of view [16], we investigated subjective speed perception in the absence of dashboard information. Interestingly, this was found to be highly correlated $(r=0.88)$ with subjective speed in real driving, and under conditions with the same velocity of optic flow, also with absolute driving speed (in both driving simulator and real road situations). Therefore, results concerning subjective speed perception in full scale driving simulators seem to be applicable to real road conditions. As we will see below, that is not true for distance perception.

In natural conditions, our sensation of depth is based upon many visual cues [34]. Some are binocular, such as DISPARITY [35], others are monocular like motion parallax. Motion parallax, generally recognized as an independent cue for perception of relative distances [36], provides robust estimates of absolute egocentric distances when combined with extra-retinal information about the observer's self-motion (see Box 2). In a recent series of experiments [37], it has been shown that the central nervous system is able to combine these two types of information to account for repetitive head movements, even when they are small (approx. $5 \mathrm{~cm}$ ). This finding suggests that the integration of these two cues is also likely to be effective for natural head movements, such as those occurring during ordinary driving.

\section{Factors affecting effectiveness of visual cues}

Under natural conditions, visual cues to depth are combined in a redundant way to elicit robust perception of 3-D space. However in a driving simulator, (1) the number of depth cues might be reduced, (2) the display parameters (e.g. image resolution, frequency, field of view) could alter the temporal and spatial depth cues, and finally (3) motion cues might be missing, or partially or poorly synchronized with visual information. Few investigators have studied the effects of these parameters on driving simulator fidelity [22], or on driver behavior [38]. So far,

\section{Box 2. Extracting egocentric distance from motion parallax}

Motion parallax is the differential image motion of two points or objects $\boldsymbol{A}$ and $\boldsymbol{B}$ due to their different distances relative to the fixation point when viewed by a laterally moving observer (Fig. I). By contrast, image motion produced by the observer's movements is ambiguous in that it specifies distance only to a scaling factor $[a-c]$. The motion of each point on the retina depends on the relative position and relative motion of the object and the observer. Recent research suggests that for natural locomotion, the nervous system uses the extra-retinal information that accompanies observer movement to calibrate the retinal image motion and infer absolute distance $[d-f]$.

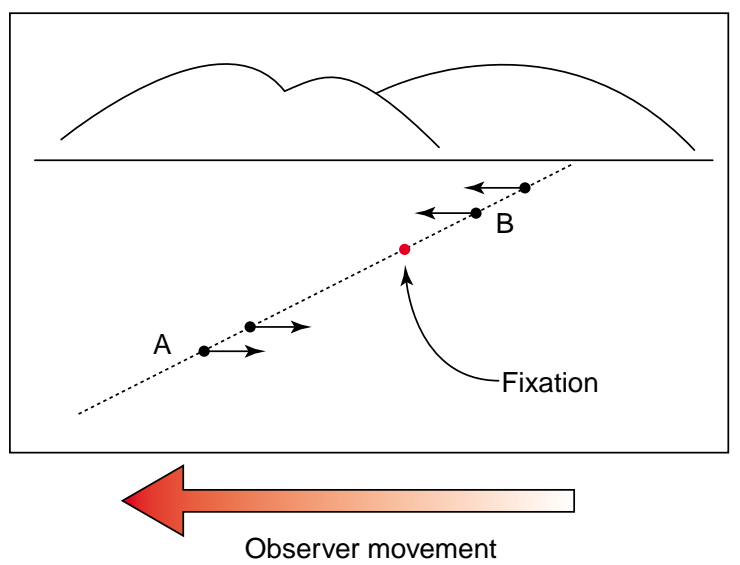

$\overline{\text { TRENDS in Cognitive Sciences }}$

Fig. I. Motion parallax. An observer moving sideways will experience a differential image motion of the two points $A$ and $B$, owing to their different distances relative to the fixation point and to the observer movement itself.

In mathematical terms, using a simplified version of the LonguetHiggins and Pradzny equations [c], one can write:

$\mathbf{U}(\mathbf{x}, \mathbf{y})=\mathbf{V} \times \mathbf{T}(\mathbf{x}, \mathbf{y}) / \mathbf{D}$

where $\mathbf{U}(\mathbf{x}, \mathbf{y})$ is the horizontal image motion for the image point $(x, y)$ (e.g. the point $A), \mathbf{T}(\mathbf{x}, \mathbf{y})$ is the normalized $3-D$ velocity (i.e. the observer movement), and $\mathbf{V}$ a coefficient defining its amplitude. If the nervous system is able to estimate such observer movement from sensory information, then the measure of the retinal motion $\mathbf{U}(\mathbf{x}, \mathbf{y})$ is sufficient to retrieve distance information as:

$\mathbf{D}=\mathbf{V} \times \mathbf{T}(\mathbf{x}, \mathbf{y}) / \mathbf{U}(\mathbf{x}, \mathbf{y})$

\section{References}

a Lee, D.N. (1980) The optic flow field. Phil. Trans. R. Soc. Lond. B Biol. Sci. 290, 169-179

b Prazdny, K. (1983) Information in the optic flow. Comp. Vis. Graph. Image Proc. 22, 235-259

c Koenderink, J.J. (1986) Optic flow. Vis. Res. 26, 161-180

d Panerai, F. et al. (2002) Contribution of extra-retinal signals to the scaling of object distance during self-motion. Percept. Psychophys. 64, 717-731

e Peh, C.H. et al. (2002) Absolute distance perception during in-depth head movement: calibrating optic flow with extra-retinal information. Vis. Res. 42, 1991-2003

f Gogel, W.C. and Tietz, J.D. (1979) A comparison of oculomotor and motion parallax cues of egocentric distance. Vis. Res. 19, $1161-1170$

there have been only limited attempts to compare perception in simulators with real driving [17-19]. One comparative study performed in a full-scale driving simulator, in which stereoscopic view and motion parallax 
were not correlated with driver self-motion, showed that drivers underestimated distances (to a leading vehicle), when compared with driving on a real road [16]. A possible interpretation of this is that the motion parallax arising from observer movement, which has been previously reported to be a crucial cue to absolute distance in near space, might be necessary for depth estimation in driving simulators. Meanwhile, the precise role of motion parallax for efficient distance estimation in driving simulation experiments remains the subject of ongoing research.

\section{Steering and vehicle speed control Heading}

Although optic flow is considered one of the most important types of visual information used for driving and for everyday locomotion [2,3], it can be ambiguous. Optic flow along the retina depends on eye and head movements [39] and different eye-head and body motion can produce very similar flow patterns. To clarify this issue, experiments were carried out to determine the role of extra-retinal information (i.e. vestibular, proprioceptive and efference copy) in disambiguating the interpretation of such complex optic flow patterns. Results showed that extra-retinal cues are crucial for correct interpretation of flow information and for heading control [40] (but also see
$[9,41])$. However, it is also important to note, that during natural locomotion (and driving) we tend to fixate points of the forthcoming trajectory. These active gaze strategies might also play an important role in heading control [42-44]. In fact, several authors have proposed that the guidance of locomotion can be achieved using purely visual egocentric direction information, without using optic flow [45-47]. Alternatively, a driver might use active gaze strategies to simplify the analysis of optic flow $[48,49]$. This latter hypothesis led to the formulation of a theoretical model of heading based on optic flow and visual egocentric direction cues [11]. These two sources of information are redundant in the driver's visual world, so, if simultaneously present, either one could enable a driver's guidance towards a given target [50] (but also see [51]). However, if road markings are missing or difficult to perceive, an optic flow-based strategy might be more robust for efficient heading control. Conversely, if the road edges are perceptible, visual egocentric direction cues (see glossary) could alone provide sufficient information.

\section{Influence of extra-visual cues in steering}

Of the different driver strategies that have been proposed for steering [52-56], a model based on visual egocentric direction cues has been the subject of recent study in

\section{Box 3. The vestibular system and its role in driving}

The vestibular system, a sensory apparatus localized bilaterally in the inner ears, detects the motion of the head and body in space [a]. It is composed of two functional parts: (1) the otolith organs (Fig. I, blue and green colored areas), and (2) the semicircular canals (Fig. I, red, orange and pink areas), which are selectively sensitive to linear and angular accelerations respectively [b]. In addition, the otoliths signal the rotation of the head relative to gravity, that is, head tilt [c], which the nervous system resolves from linear acceleration by means of internal models [d]. Normal functioning of this system is essential in many types of sensori-motor processes (e.g. compensatory eye movements,

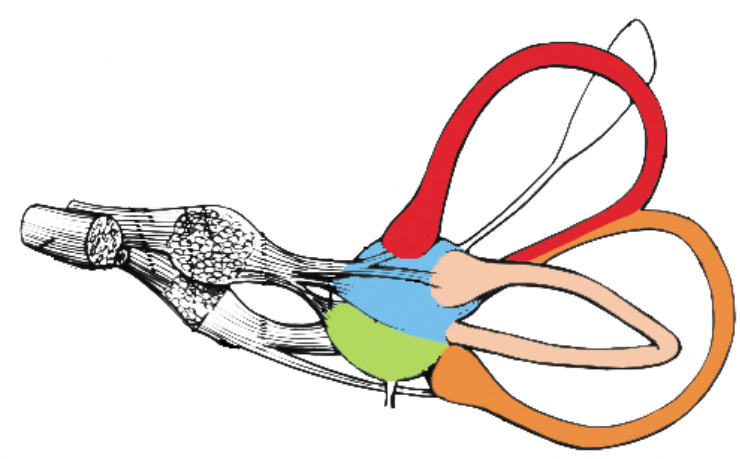

$\overline{T R E N D S ~ i n ~ C o g n i t i v e ~ S c i e n c e s ~}$

Fig. I. The vestibular system and its measurement principles. The three semicircular canals (red, orange and pink) are filled with a viscous liquid, the endolymph. When the head is moved, the liquid exerts a pressure on the cupula, a specialized structure localized at the end of each canal. Pressure stimuli is transformed into nerve discharge, encoding the angular acceleration of the head. Similarly, the otolith receptors (blus and green), which are composed of a mass of crystals floating in the endolymph, encode both linear acceleration and tilt of the head. postural control). Furthermore, vestibular information has important roles in perceptual tasks such as egomotion estimation [e]. More recently, vestibular information was shown to disambiguate the interpretation of dynamic visual information experienced simultaneously during observer's movement [f]. In driving simulation, the absence of vestibular information has been reported to increase steering reaction times to external movement perturbations [g], and also to decrease safety margins in the control of lateral acceleration in curve driving [h]. In real driving, improper signals from disordered vestibular organs were reported to determine inappropriate steering adjustment [i]. Moreover, the presence of vestibular information in driving simulators seems important for it influences the perception of illusory self-tilt and illusory self-motion [j].

\section{References}

a Berthoz, A. (2000) The Brain's Sense of Movement, Harvard University Press

b Goldberg, J.M. and Fernandez, C. (1975) Responses of peripheral vestibular neurons to angular and linear acceleration in the squirrel monkey. Acta Otolaryngol. 80, 101-110

c Seidmann, S.H. et al. (1998) Tilt perception during dynamic linear acceleration. Exp. Brain Res. 119, 307-314

d Merfeld, D.M. et al. (1999) Humans use internal models to estimate gravity and linear acceleration. Nature $398,615-618$

e Berthoz, A. et al. (1995) Spatial memory of body linear displacement: what is being stored? Science 269, 95-98

f Wexler, M. et al. (2001) The stationarity hypothesis: an allocentric criterion in visual perception. Vis. Res. 41, 3023-3037

g Wierville, W.W. et al. (1983) Driver steering reaction time to abrupt onset crosswind, as measured in a moving-base driving simulator. Hum. Factors 25, 103-116

h Reymond, G. et al. (2001) Role of lateral acceleration in curve driving: driver model and experiments on a real vehicle and a driving simulator. Hum. Factors 43, 483-495

i Page, N.G. and Gresty, M.A. (1985) Motorist's vestibular disorientation syndrome. J. Neurol. Neurosurg. Psychiatry 48, 729-735

$\mathrm{j}$ Groen, E.L. et al. (1999) Influence of body roll on visually induced sensation of self-tilt and rotation. Perception 28, 287-297 


\section{Box 4. Questions for future research}

- Although driving is generally considered to be visually guided, what is the role of vestibular information in longitudinal and lateral vehicle control? Moreover, what is the precise role of driver action (steering and speed control) in self-motion perception and in the integration of visuo-vestibular cues (driver vs passenger perception)?

- As cues to absolute distance in near space, the effectiveness of stereopsis and motion parallax from self-movement is wellestablished from experiments inside the vehicle or in its close vicinity. By contrast, their effectiveness more distally, in the driver's case for observation of other vehicles or markings on the road, is more controversial. To what extent would their inclusion in driving simulators increase driver performance in these experiments?

- What is the influence of cognitive factors, such as safety margins, various driving strategies or internal models of driver performance in driving simulation experiments (e.g. braking, safety distances, curve driving)?

driving simulation experiments [12]. However, earlier studies have shown that the absence of physical motion in a driving simulator modifies the driver's reactions [19]. Moreover, computational models of self-motion perception [57] and studies performed on a moving-base driving simulator indicate that driver's control strategies on curved roads make use not only of visual, but also of extra-visual information, such as vestibular (see Box 3) and proprioceptive cues [14,58]. So, on the basis of models proposed in earlier studies [58,59], it has been suggested that these cues are used by the driver to control steering and regulate speed. Indeed, experiments performed in moving-based driving simulators show that drivers take wider turns when lateral cues are present, compared to the way they steer under conditions in which only visual information is available [60]. The role of vestibular cues in perception of natural self-motion have been well studied [61-66]. It appears that to completely understand vehicle driving, the precise role of vestibular and other HAPTIC and KINAESTHETIC cues in steering and speed control, especially when driving on curved roads, must now be investigated further in motion-based driving simulation experiments (see also Box 4).

\section{Conclusion}

Driving simulation can provide important information for vehicle design and thanks to its inter-disciplinary nature, it can foster basic and applied research, opening new directions of investigation in the study of multi-sensory integration for self-motion perception. Driving simulation experiments have led to novel interpretations of the role of egocentric direction, and vestibular cues in steering and speed perception respectively. For accurate perception of vehicle speeds and distances, simulation studies recommend the use of a large field of view, and the rendering of motion parallax due to observer's self-motion. Such results, reinforced by the recent psychophysical studies reviewed here, demonstrate how driving simulators can lead to a more thorough understanding of human perception and control of self-motion, especially when speeds and accelerations are higher than in natural locomotion.
Finally, such applied psychophysics research is of direct benefit to society, most notably in road safety studies.

\section{Acknowledgements}

The authors would like to thank Prof. Alain Berthoz for his continuous help in enabling a strong research cooperation between Renault and the CNRS in the field of driving simulation, as well as Dr S. Wiener and the anonymous referees for comments on previous versions of this manuscript. Finally, we also thank France Maloumian for her help in preparing Fig. I, Box 3.

\section{References}

1 Gibson, J.J. and Crooks, L.E. (1938) A theoretical field-analysis of automobile-driving. Am. J. Psychol. 51, 453-471

2 Gibson, J.J. (1950) The Perception of the Visual World, Houghton Mifflin

3 Lappe, M. et al. (1999) Perception of self-motion from visual flow. Trends Cogn. Sci. 3, 329-336

4 Gray, R. and Regan, D. (2000) Visually guided collision avoidance and collision achievement. Trends Cogn. Sci. 4, 99-107

5 Lee, D.N. (1980) The optic flow field. Phil. Trans. R. Soc. Lond. B Biol. Sci. 290, 169-179

6 Lee, D.N. (1976) A theory of visual control of braking based on information about time-to-collision. Perception 5, 437-459

7 Tresilian, J.R. (1999) Visually timed action: time out for 'tau'? Trends Cogn. Sci. 3, 301-310

8 Warren, W.H. Jr et al. (1991) On the sufficiency of the velocity field for perception of heading. Biol. Cybern. 65, 311-320

$9 \mathrm{Li}, \mathrm{L}$. and Warren, W.H. Jr (2002) Retinal flow is sufficient for steering during observer rotation. Psychol. Sci. 13, 485-491

10 Rushton, S.K. et al. (1998) Guidance of locomotion on foot uses perceived target location rather than optic flow. Curr. Biol. 8, 1191-1194

11 Wann, J. and Land, M. (2000) Steering with or without the flow: is the retrieval of heading necessary? Trends Cogn. Sci. 4, 319-324

12 Rushton, S.K. and Salvucci, D.D. (2001) An egocentric account of the visual guidance of locomotion. Trends Cogn. Sci. 5, 6-7

13 Nordmark, S. et al. (1984) Moving base driving simulator with wide angle visual system. SAE Technical Paper Series 845100, Warrendale, PA: Society of Automobile Engineers

14 Reymond, G. et al. (2001) Role of lateral acceleration in curve driving: driver model and experiments on a real vehicle and a driving simulator. Hum. Factors 43, 483-495

15 Drosdol, J. et al. (1985) The Daimler-Benz driving simulator, a tool for vehicle development. SAE Technical Paper. 940227 Warrendale, PA: Society of Automobile Engineers

16 Panerai, F. et al. (2001) Speed and safety distance control in truck driving: comparison of simulation and real-world environment. Proc. Driving Simulation Conf. DSC 2000, Paris, France

17 Burns, P.C. et al. (1999) Intersection between driving in reality and virtual reality (VR). Proc. Driving Simulation Conf. DSC 1999, Paris, France

18 Boer, E.R. et al. (2000) Experiencing the same road twice: a driver comparison between simulation and reality. Proc. Driving Simul. Conf. Paris, France

19 Wierwille, W.W. et al. (1983) Driver steering reaction time to abruptonset crosswinds, as measured in a moving-base driving simulator. Hum. Factors 25, 103-116

20 Cavallo, V. et al. (2001) Distance perception of vehicle rear lights in fog. Hum. Factors 43, 442-451

21 Snowden, R.J. et al. (1998) Speed perception fogs up as visibility drops. Nature 392,450

22 Jamson, H. (2000) Driving simulation validity: issues of field of view and resolution. Proc. Driving Simul. Conf. DSC 2000, Paris, France

23 Loomis, J.M. (2001) Looking down is looking up. Nature 414, 155-156

24 Ooi, T.L. et al. (2001) Distance determined by the angular declination below the horizon. Nature 414, 197-200

25 Beusmans, J.M. (1998) Optic flow and the metric of the visual ground plane. Vision Res. 38, 1153-1170 
26 Cavallo, V. and Laurent, M. (1988) Visual information and skill level in time-to-collision estimation. Perception 17, 623-632

27 Bremmer, F. and Lappe, M. (1999) The use of optical velocities for distance discrimination and reproduction during visually simulated self motion. Exp. Brain Res. 127, 33-42

28 Redlick, F.P. et al. (2001) Humans can use optic flow to estimate distance of travel. Vision Res. 41, 213-219

29 Blakemore, M.R. and Snowden, R.J. (1999) The effect of contrast upon perceived speed: a general phenomenon? Perception 28, 33-48

30 Blakemore, M.R. and Snowden, R.J. (2000) Textured backgrounds alter perceived speed. Vision Res. 40, 629-638

31 Takeuchi, T. and De, V. (2000) Velocity discrimination in scotopic vision. Vision Res. 40, 2011-2024

32 Snowden, R.J. et al. (1998) Speed perception fogs up as visibility drops. Nature 392, 450

33 Gegenfurtner, K.R. et al. (1999) Seeing movement in the dark. Nature $398,475-476$

34 Cutting, J.E. et al. (1995) Perceiving layout and knowing distances: the integration, relative potency, and contextual use of different information about depth. Perception of Space and Motion (Epstein, W., Rogers, S., et al. eds), pp. 69-117, Academic Press

35 Howard, I.P. and Rogers, B.J. (1995) Binocular Vision and Stereopsis, Oxford University Press

36 Rogers, B. and Graham, M. (1979) Motion parallax as an independent cue for depth perception. Perception 8, 125-134

37 Panerai, F. et al. (2002) Contribution of extra-retinal signals to the scaling of object distance during self-motion. Percept. Psychophys. 64, $717-731$

38 Chatziastros, A. et al. (1999) In The Effect of Field of View and Surface Texture on Driver Steering Performance (Gale, A.E. et al., eds), pp. 253-259, Elsevier

39 Regan, D. and Beverley, K.I. (1982) How do we avoid confounding the direction we are looking and the direction we are moving? Science 215 , 194-196

40 Crowell, J.A. et al. (1998) Visual self-motion perception during head turns. Nat. Neurosci. 1, 732-737

$41 \mathrm{Li}, \mathrm{L}$. and Warren, W.H. Jr (2000) Perception of heading during rotation: sufficiency of dense motion parallax and reference objects. Vision Res. 40, 3873-3894

42 Land, M.F. (1992) Predictable eye-head coordination during driving. Nature 359, 318-320

43 Land, M.F. and Lee, D.N. (1994) Where we look when we steer. Nature $369,742-744$

44 Land, M. and Horwood, J. (1995) Which parts of the road guide steering? Nature 377, 339-340

45 Rushton, S.K. et al. (1998) Guidance of locomotion on foot uses perceived target location rather than optic flow. Curr. Biol. 8, 1191-1194

46 Harris, J.M. and Rogers, B.J. (1999) Going against the flow. Trends Cogn. Sci. 3, 449-450
47 Rogers, B.J. and Dalton, C. (1999) The role of (i) perceived direction and (ii) optic flow in the control of locomotion and for estimating the point of impact. Invest. Ophthalmol. Vis. Sci. 40, S746

48 Kim, N.G. and Turvey, M.T. (1999) Eye-movements and a rule for perceiving direction of heading. Ecol. Psychol. 11, 233-248

49 Wann, J.P. and Swapp, D.K. (2000) Why you should look where you are going. Nat. Neurosci. 3, 647-648

50 Harris, J.M. (2001) The future of flow? Trends Cogn. Sci. 5, 7-8

51 Harris, J.M. and Bonas, W. (2002) Optic flow and scene structure do not always contribute to the control of human walking. Vision Res. 42, 1619-1626

52 Donges, E. (1978) A two-level model of driver steering behavior. Hum. Factors 20, 691-707

53 Godthelp, H. (1986) Vehicle control during curve driving. Hum. Factors 28, 211-221

54 Beall, A.C. and Loomis, J.M. (1996) Visual control of steering without course information. Perception 25, 481-494

55 Hildreth, E.C. et al. (2000) From vision to action: experiments and models of steering control during driving. J. Exp. Psychol. Hum. Percept. Perform. 26, 1106-1132

56 Wallis, G. et al. (2002) An unexpected role for visual feedback in vehicle steering control. Curr. Biol. 12, 295-299

57 Reymond, G. et al. (2002) Visuovestibular perception of self-motion modeled as a dynamic optimization process. Biol. Cybern. 87, 301-314

58 Van Winsum, W. and Godthelp, H. (1996) Speed choice and steering behavior in curve driving. Hum. Factors 38, 434-441

59 Godthelp, H. et al. (1984) The development of a time-related measure to describe driving strategy. Hum. Factors 26, 257-268

60 Siegler, I. et al. (2001) Sensorimotor integration in a driving simulator: contribution of motion cueing in elementary driving tasks. Proc. Driving Simul. Conf. DSC 2001, Sophia Antipolis, Nice, France

61 Wertheim, A.H. (1994) Motion perception during self-motion: the direct versus inferential controversy revisited. Behav. Brain Sci. 17, 293-355

62 Harris, L.R. et al. (2000) Visual and non-visual cues in the perception of linear self-motion. Exp. Brain Res. 135, 12-21

63 Harris, L.R. et al. (2002) Simulating self motion I: cues for the perception of motion. Virtual Reality 6, 75-85

64 Israel, I. et al. (1997) Spatial memory and path integration studied by self-driven passive linear displacement. I. Basic properties. J. Neurophysiol. 77, 3180-3192

65 Ivanenko, Y. et al. (1997) Spatial orientation in humans: perception of angular whole-body displacements in two-dimensional trajectories. Exp. Brain Res. 117, 419-427

66 Berthoz, A. et al. (1982) Linear self-motion perception. In Tutorials on Motion Perception (Wertheim, A.H. et al., eds), pp. 157-199, Plenum Press

\section{Managing your references and BioMedNet Reviews}

Did you know that you can now download selected search results from BioMedNet Reviews directly into your chosen reference-managing software? After performing a search, simply click to select the articles you are interested in, choose the format required (e.g. EndNote 3.1) and the bibliographic details, abstract and link to the full-text will download into your desktop reference manager database.

BioMedNet Reviews is available on institute-wide subscription. If you do not have access to the full-text articles in BioMedNet Reviews, ask your librarian to contact reviews.subscribe@biomednet.com 